\title{
Hierarchical Domain Decomposition Method for Devices including Moving Bodies
}

\author{
Shin-ichiro Sugimoto ${ }^{1 *}$, Masao Ogino ${ }^{2}$, Hiroshi Kanayama $^{3}$, Amane Takei $^{4}$ \\ ${ }^{1}$ Department of Mechanical Engineering, Faculty of Engineering, \\ Tokyo University of Science, Suwa \\ ${ }^{2}$ Information Technology Center, Nagoya University \\ ${ }^{3}$ Department of Mathematical and Physical Sciences, Faculty of Science, \\ Japan Women's University \\ ${ }^{4}$ Department of Electrical and System Engineering, Faculty of Engineering, \\ University of Miyazaki \\ *sugimoto@rs.tus.ac.jp
}

Received: July 11, 2017; Accepted: January 31, 2018; Published: March 31, 2018

\begin{abstract}
This paper deals with three-dimensional non-steady eddy current analysis of a rotating machine as an example of a parallel finite element analysis including moving bodies. In general, high efficiency in parallel computing with a moving body is difficult to achieve. The hierarchical domain decomposition method (HDDM) is known as an efficient parallel finite element method. However, in cases that involve a moving body, the HDDM with static domain decomposition has not attained sufficient parallel efficiency. Moreover, the cost of dynamic domain decomposition is substantial. In this paper, we propose a new domain decomposition technique for the HDDM that enables us to achieve efficient scalability on massively parallel computers. Our method's parallel efficiency has been $93.3 \%$ on 96 computer nodes (1,536 cores) of the Oakleaf-FX supercomputer. Furthermore, an induction motor model with a seven million degrees of freedom mesh whose solution by conventional sequential computation requires more than a month has been successfully solved in approximately 1.60 hours using the proposed method.
\end{abstract}

Keywords: Parallel computing, Hierarchical domain decomposition method, Rotating machine, Finite element analysis

\section{Introduction}

There are many devices including moving bodies, and rotating machines such as electric generators or motors are representative. Such devices have become essential in 


\section{Journal of Advanced Simulation in Science and Engineering}

our lives; thus, the development of an efficient rotating machine has become necessary to reduce cost and environmental loading. Designing rotating machines includes electromagnetic analysis, which takes substantial time; thus, methods for reducing the time steps, such as time periodic explicit error correction (TP-EEC) [1, 2] and time differential correction (TDC) [3], and for reducing the computation time, such as the parallel iterative method $[4,5]$, have been proposed. However, these methods cannot utilize massively parallel computers efficiently, and still take substantial time to analyze a rotating machine. However, for non-linear magnetostatic problems, time-harmonic eddy current problems, and high-frequency electromagnetic problems, problems with billions of degrees of freedom (DOFs) have been solved efficiently on the massively parallel computers using the hierarchical domain decomposition method (HDDM) [6-8]. Nevertheless, in cases that involve a moving body, the HDDM with static domain decomposition has not attained sufficient parallel efficiency. Moreover, the cost of dynamic domain decomposition is substantial. In this paper, we propose a new domain decomposition technique for the HDDM for analyzing devices including moving bodies efficiently on massively parallel computers. Then, we confirm the computational efficiency of our proposed method through an analysis of a simplified induction motor model.

\section{HDDM}

\subsection{Outline}
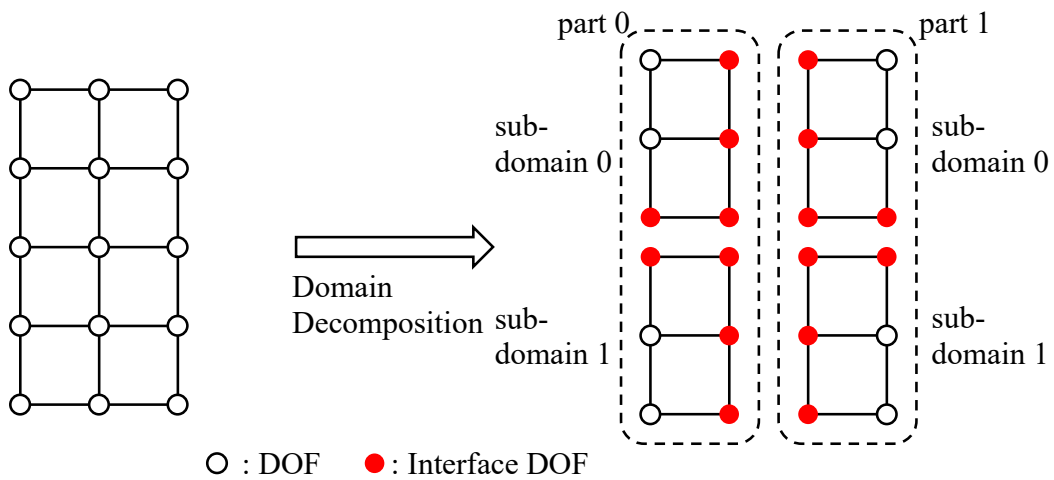

Fig. 1. Hierarchical domain decomposition.

The HDDM is a method for efficiently implementing domain decomposition [9-11] in massively parallel environments and is well known as an efficient parallel computing method for large-scale finite element analysis. Figure 1 shows a pattern diagram of domain decomposition in the HDDM. In this paper, to make visual understanding easier, we draw a similar sort of pattern diagram using quadrilateral elements with the DOFs at the nodes, instead of the customary piecewise linear tetrahedral and edge elements. 


\section{Journal of Advanced Simulation in Science and Engineering}

In the HDDM, an original domain $\Omega$ is divided without overlapping elements. Furthermore, the original domain is hierarchically divided into parts, which are further decomposed into smaller domains called subdomains.

$$
\Omega=\bigcup_{i=0}^{N_{p}-1} \bigcup_{j=0}^{N_{S}-1} \Omega^{(i, j)}
$$

where $\Omega^{(i, j)}$ denotes the subdomain $j$ in the part $i$, the superscript $(i, j)$ corresponds to the subdomain $\Omega^{(i, j)} . N_{p}$ and $N_{s}$ are the number of parts and that of subdomains in each part, respectively. $N_{s}$ is equal for all parts. In Fig. 1, the original domain is first divided into two parts, and then further decomposed into two subdomains within each part $\left(N_{P}=N_{S}=2\right)$. The red solid circles in the figure are the DOFs shared between parts and subdomains, which are called interface DOFs.

Let us denote the finite element equation in $\Omega$ by the matrix form

$$
K u=f,
$$

where $\boldsymbol{K}$ denotes the symmetric matrix, $\boldsymbol{u}$ the DOFs, and $\boldsymbol{f}$ the known vector. After the domain decomposition, by reordering, the linear system ( 2 ) is rewritten by

$$
\left[\begin{array}{ll}
K_{I I} & K_{I B} \\
K_{I B}^{T} & K_{B B}
\end{array}\right]\left[\begin{array}{l}
u_{I} \\
u_{B}
\end{array}\right]=\left[\begin{array}{l}
f_{I} \\
f_{B}
\end{array}\right]
$$

where

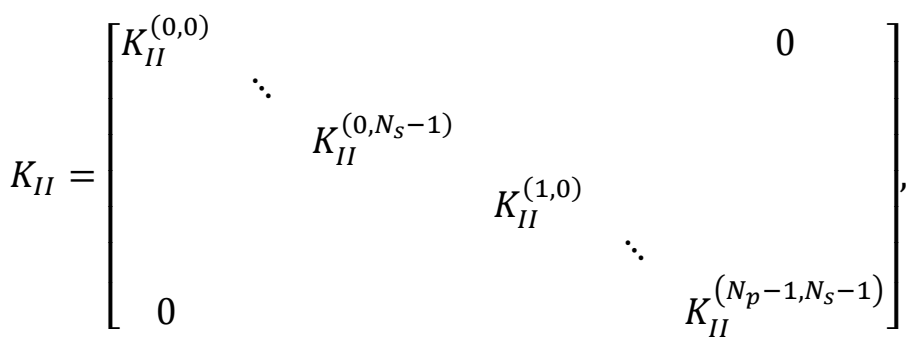

$$
\begin{aligned}
& K_{I B}=\left[\begin{array}{c}
K_{I B}^{(0,0)} R_{B}^{(0,0)} \\
\vdots \\
K_{I B}^{\left(0, N_{S}-1\right)} R_{B}^{\left(0, N_{s}-1\right)} \\
K_{I B}^{(1,0)} R_{B}^{(1,0)} \\
\vdots \\
K_{I B}^{\left(N_{p}-1, N_{s}-1\right)} R_{B}^{\left(N_{p}-1, N_{s}-1\right)}
\end{array}\right], \quad K_{B B}=\sum_{i=0}^{N_{p}-1} \sum_{j=0}^{N_{s}-1} R_{B}^{(i, j) T} K_{B B}^{(i, j)} R_{B}^{(i, j)}, \\
& f_{i}=\sum_{i=0}^{N_{p}-1} \sum_{j=0}^{N_{S}-1} R_{I}^{(i, j) T} f_{I}^{(i, j)}, \quad f_{B}=\sum_{i=0}^{N_{p}-1} \sum_{j=0}^{N_{S}-1} R_{B}^{(i, j) T} f_{B}^{(i, j)},
\end{aligned}
$$

where the subscripts $I$ and $B$ correspond to DOFs in the interior of subdomains and the interface DOFs, respectively. $R_{I}^{(i, j)}$ and $R_{B}^{(i, j)}$ are the Boolean matrices to map the global DOFs to local DOFs. DOFs are reduced statically, and the interface problem is written by 


$$
S u_{B}=g,
$$

where

$$
\begin{aligned}
& S=\sum_{i=0}^{N_{p}-1} \sum_{j=0}^{N_{s}-1} R_{B}^{(i, j) T}\left(K_{B B}^{(i, j)}-K_{I B}^{(i, j) T}\left(K_{I I}^{(i, j)}\right)^{\dagger} K_{I B}^{(i, j)}\right) R_{B}^{(i, j)}, \\
& g=\sum_{i=0}^{N_{p}-1} \sum_{j=0}^{N_{S}-1} R_{B}^{(i, j) T}\left(f_{B}^{(i, j)}-K_{I B}^{(i, j) T}\left(K_{I I}^{(i, j)}\right)^{\dagger} f_{I}^{(i, j)}\right),
\end{aligned}
$$

where $S$ is the Schur complement matrix, $\left(K_{I I}^{(i, j)}\right)^{\dagger}$ the generalized inverse of $K_{I I}^{(i, j)}$. In the HDDM, we first solve ( 4 ) by using an iterative method such as the conjugate gradient (CG) method in the parallel environments. Then, we solve the finite element equation ( 5 ) in the subdomains by considering the interface DOFs as Dirichlet conditions.

$$
\begin{aligned}
K_{I I}^{(i, j)} u_{I}^{(i, j)}=f_{I}^{(i, j)}-K_{I B}^{(i, j)} R_{B}^{(i, j)} u_{B}, & \\
& \quad i=0, \ldots, N_{p}-1, j=0, \ldots, N_{S}-1 .
\end{aligned}
$$

\subsection{Communication tables in the HDDM}

In this section, with Fig. 1 as an example, the communication tables in the HDDM are described based on implementation in the ADVENTURE_Magnetic [12].

In the HDDM, each message passing interface (MPI) process handles one part. Therefore, it is necessary to perform interprocess communication concerning the interface DOFs shared between parts. In the HDDM, parts and MPI processes correspond one to one; thus, to simplify the explanation, we associate the zeroth MPI process with part 0 and the first MPI process with part 1.

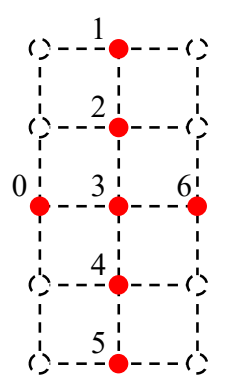

(a) Interface DOFs

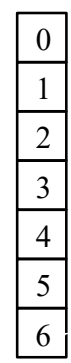

(b) Vector of $u_{B}$

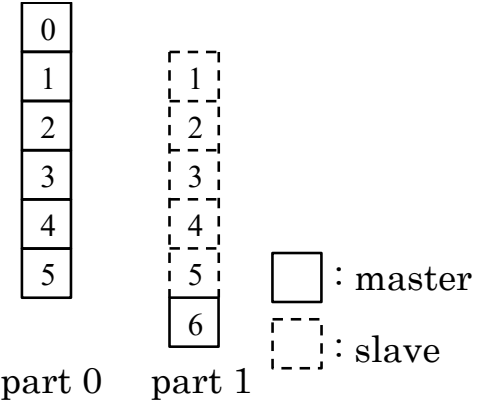

(c) Memory spaces in each part

Fig. 2. Memory spaces for interface DOFs.

In the case of Fig. 1, there are seven interface DOFs (Fig. 2 (a)); thus, $u_{B}$ is the vector with seven elements (Fig. 2 (b)). However, since the original domain is decomposed, the vector data are stored in each MPI processes distributedly. Furthermore, the interface DOF shared between the parts has memory space in each part. Here, there are multiple memory spaces for 
the same interface DOF; thus, one memory space is to be the master. In the ADVENTURE_Magnetic, the memory space of the part with the smallest part number among the parts having memory spaces of the same interface DOF is defined as the master. Interface DOFs " 1 , $2,3,4,5$ " are shared between part 0 and 1 ; thus, memory spaces of part 0 are to be their masters, and those of part 1 are their slaves (Fig. 2 (c)). In each part, interface DOF having master memory space is called "primary interface DOF", and that having slave one "dependent interface DOF". In part 0, all interface DOFs are primary interface DOFs. In part 1, “1, 2, 3, 4, 5" are dependent interface DOFs, and " 6 " is the primary interface DOF. Interprocess communication concerning the interface DOFs shared between parts is performed during matrix-vector product operations of the Schur complement matrix. First, independent computations are performed in each part. Next, the results are sent from part 1 having slave memory spaces to part 0 having master memory spaces. After performing necessary computations in part 0 , the results are sent to part 1 , and they are copied to the slave memory spaces of part 1 .

To perform interprocess communications, a set of communication tables is made. First, the local serial numbers of the interface DOFs are assigned in each part. Serial numbers are assigned in order of the dependent interface DOFs and the primary interface DOFs. For example, the serial numbers of the interface DOFs in each part are assigned as shown in Fig. 3. In part 1, "1, 2, 3, 4, 5" in Fig. 2 (a) are dependent interface DOFs; thus, the local serial numbers are assigned to them, firstly. For " $0,1,2,4,5$ " of part 0 and " $0,1,2,3,4$ " of part 1 , the set of communication tables is as shown in Fig. 4. Here, "part 0 " and "part 1" indicate communication partners, respectively, and the numerals following "." indicate the numbers of interface DOFs communicating with the partner. For example, in the communication table of part 0 , since part 0 does not need to communicate with part 0 , the number of interface DOFs next to "part 0:" is 0 . In addition, since five interface DOFs are shared with part 1 , the number of interface DOFs next to "part 1:" is five. When the number of interface DOFs is one or more, the next line lists the local serial numbers within the part of the interface DOFs shared with the communication partner. Here, 3 in part 0 and 5 in part 1 are shared only between subdomains in the same part; thus, they are not included in the communication tables. Then, interprocess communication is performed based on the set of communication tables.

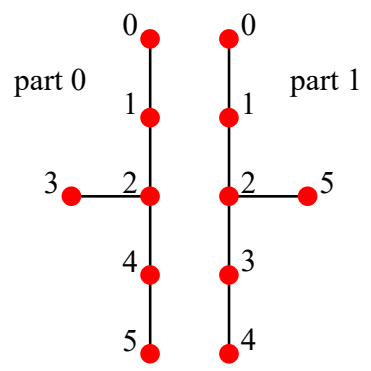

Fig. 3. Serial numbers of interface DOFs in each part.

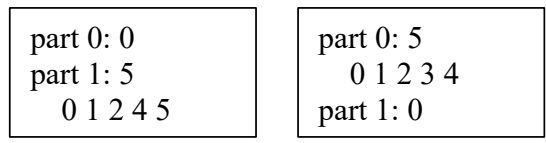

Fig. 4. Set of communication tables (Left: for part 0, Right: for part 1). 


\section{Treatment of moving bodies in the HDDM}

\subsection{Outline}

In the electromagnetic field analysis of rotating machines, the moving body (rotor) moves at each time step; thus, the connection relation of the meshes between the moving body and the other stationary parts (such as the stator) changes. Therefore, in many cases, meshes are generated with the element surfaces coinciding even if the moving body moves, as shown in Fig. 5. Here, the purple solid circles on the connection surface indicate that the DOFs corresponding to the stationary and moving bodies are treated as the same DOF. In this paper as well, we will follow this approach and consider efficient implementation in the HDDM.

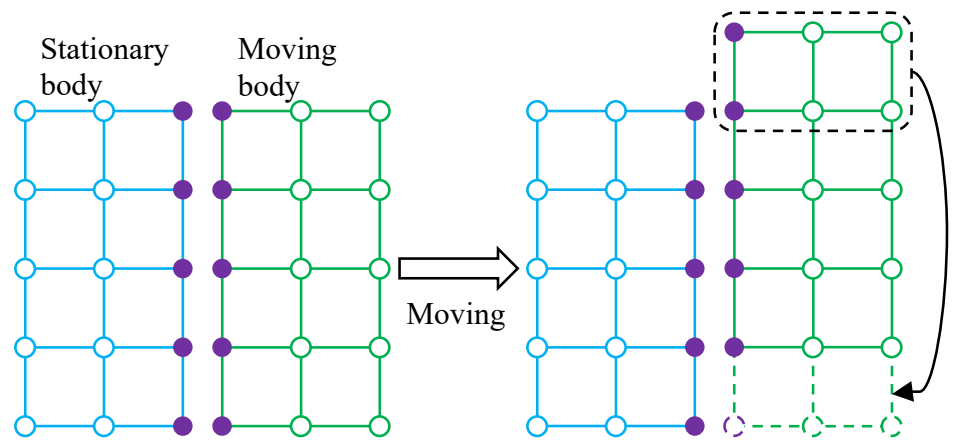

: DOF on connection surface $\bigcirc$ : DOF in stationary body $\bigcirc$ : DOF in moving body

Fig. 5. Stationary and moving bodies.

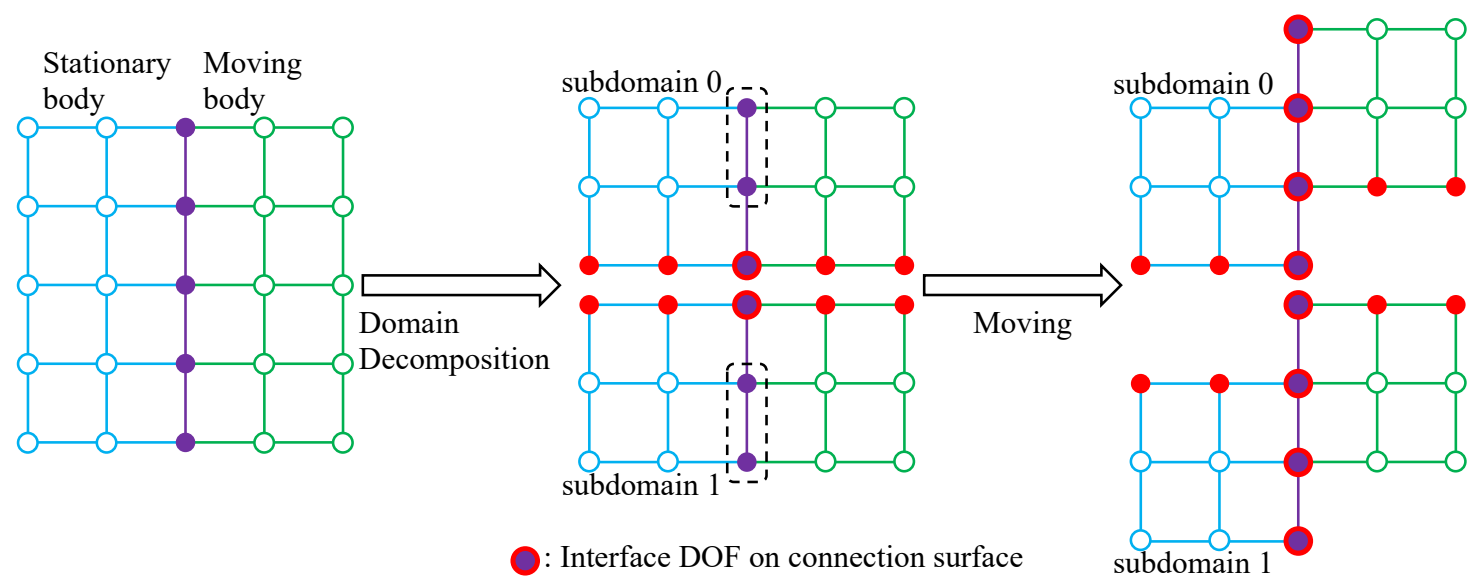

Fig. 6. Moving of combined mesh.

We assume that the combined mesh of the stationary and moving bodies is decomposed without any intention. In the case of domain decomposition of the combined mesh on the left in Fig. 6, the DOFs on the connection surface might be located inside the subdomain, as shown in the middle of Fig. 6. In this case, the DOFs that are originally not the interface DOFs surrounded by the dotted line change to interface DOFs, as shown on the right in Fig. 6, as a result of the movement; this complicates the interprocess communication. Furthermore, degrees of freedom of the subdomain also change in accordance with the movement (in this case, they 
change from 15 to 16), and the processing inside the subdomain also becomes complicated. Therefore, it is considered that the implementation complexity increases and the computation efficiency decreases.

In order to prevent the DOFs on the connection surface from being located inside the subdomain, meshes of stationary and moving bodies are decomposed independently (Fig. 7). The connection surface appears on the surface of the subdomains; thus, the DOFs on the connection surface are treated as interface DOFs shared between parts. Here, a set of communication tables relating to the original interface DOFs and sets of communication tables of the DOFs on the connection surface changing with time evolution are prepared separately. Then, they are used in combination at each time step.
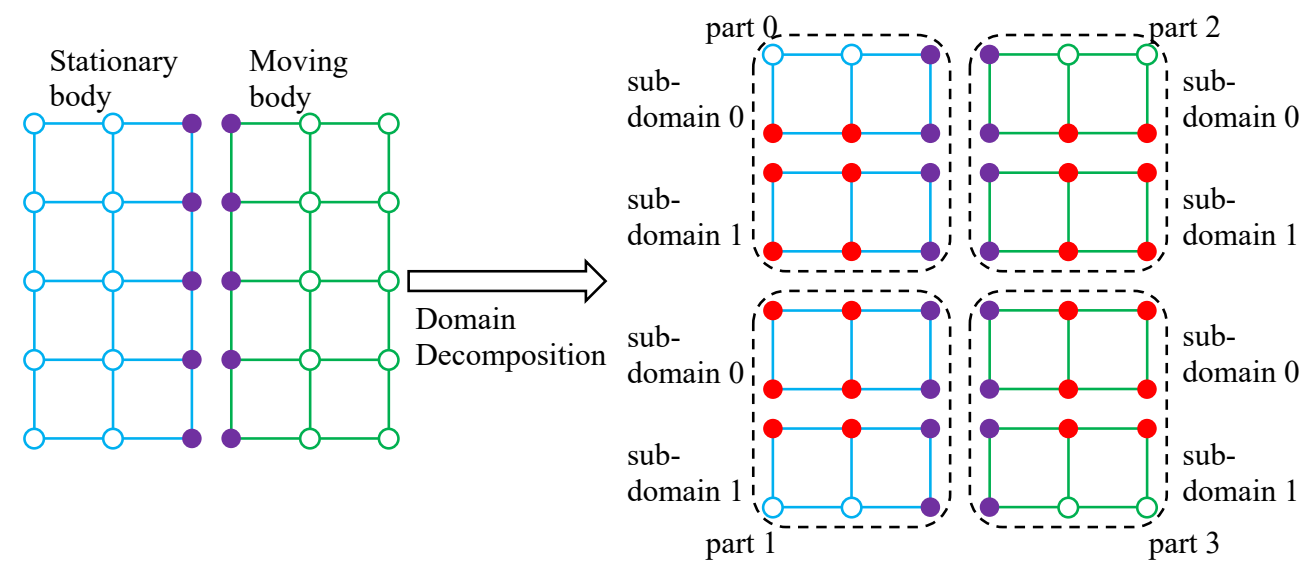

Fig. 7. Independent domain decomposition of stationary and moving bodies.

\subsection{Communication tables of a device including moving bodies}

In this section, communication tables in the case in which the meshes of the stationary and moving bodies are decomposed independently will be described, based on Fig. 7 .

In Fig. 7, the total number of processes is four, and two processes are allocated to the stationary and moving bodies, respectively. Next, each body is first divided into two parts, and then further decomposed into two subdomains within each part. Part numbers are consecutive starting from the stationary body. The red solid circles in the figure are the interface DOFs caused by the decomposition, but the DOFs on the connection surface are excluded.

The local serial numbers in each part are consecutive starting from the original interface DOFs (red solid circles) both in the dependent interface DOFs, and the primary interface DOFs; thus, serial numbers are assigned in the order of the dependent interface DOFs of red solid circles, the dependent interface DOFs of the DOFs on the connection surface (purple solid circles), the primary interface DOFs of red solid circles, and the primary interface DOFs of purple solid circles. As a result, the local serial numbers of the interface DOFs in each part are as shown in Fig. 8. Here, the interface DOFs surrounded by the dotted line are the dependent interface DOFs.

In part 0 , all interface DOFs are the primary interface DOFs; thus, red solid circles have serial numbers 0 to 3 , and purple solid circles have serial numbers 4 to 6 . In part 1 , interface DOFs shared with part 0 are the dependent interface DOFs, and the others are the primary 


\section{Journal of Advanced Simulation in Science and Engineering}

interface DOFs; thus, the red solid circles that are the dependent interface DOFs have serial numbers 0 and 1 , and the purple solid circle that is the dependent interface DOF has serial number 2. Furthermore, the red solid circles that are the primary interface DOFs have serial numbers 3 and 4, and the purple solid circles that are the primary interface DOFs have serial numbers 5 and 6 .

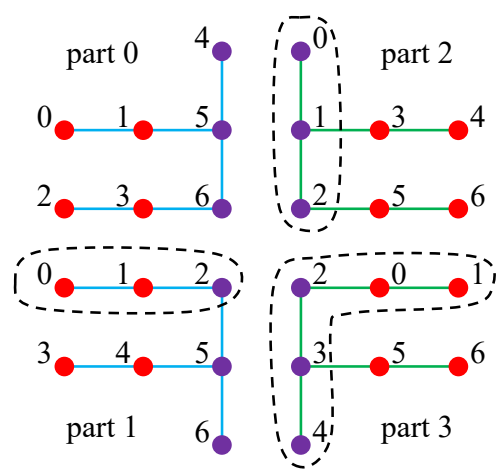

Fig. 8. Serial numbers of interface DOFs in each part of the rotating machine.

\begin{tabular}{|l|}
\hline part $0: 0$ \\
part 1: 2 \\
23 \\
part 2: 0 \\
part 3: 0 \\
\hline
\end{tabular}

part 0

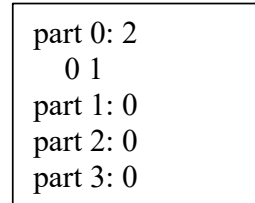

part 1

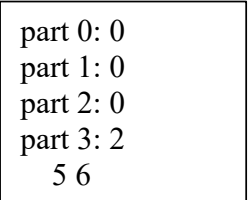

part 2

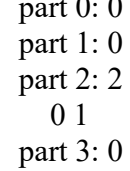

01

part 3:0

part 3

Fig. 9. Set of communication tables (red solid circles).

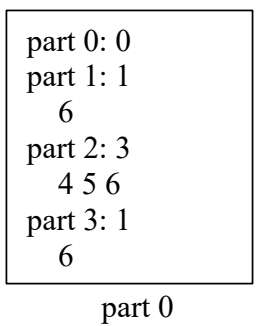

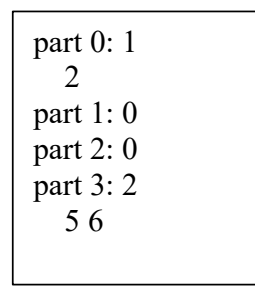

part 1

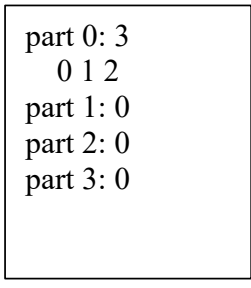

part 2

\begin{tabular}{|l|}
\hline part $0: 1$ \\
2 \\
part $1: 2$ \\
34 \\
part 2:0 \\
part 3: 0 \\
\hline
\end{tabular}

part 3

Fig. 10. Set of communication tables of step 0 (purple solid circles).

In the moving body, all the purple solid circles are shared with parts of the stationary body. Here, since all the part numbers of the moving body are larger than all the part numbers of the stationary body, there is no purple solid circle that is the primary interface DOF in the moving body. In part 2, the interface DOFs shared with parts 0 and 1 are the dependent interface DOFs, and the others are the primary interface DOFs. There is no red solid circle that is the dependent interface DOF; thus, the purple solid circles that are the dependent interface DOFs have serial numbers 0 to 2, and the red solid circles that are the primary interface DOFs have serial numbers 3 to 6 . In part 3, the interface DOFs shared with parts 0 to 2 are the dependent interface DOFs, and the others are the primary interface DOFs. The red solid circles that are the dependent interface DOFs have serial numbers 0 and 1 , and the purple solid circles that are the dependent interface DOFs have serial numbers 2 to 4 . Then, the red solid circles that are the primary interface DOFs have serial numbers 5 and 6 . 


\section{Journal of Advanced Simulation in Science and Engineering}

Subsequently, two types of sets of communication tables are created. One is a set of communication tables that relates only to red solid circles. This is an unchanged set regardless of the movement of the moving body (i.e. time evolution). The other is a set of communication tables related to purple solid circles. This changes with the movement; thus, as many are created as the number of connection patterns of the meshes. Figures 9 and 10 show the set of communication tables (red solid circles) and the set of communication tables of step 0 (purple solid circles) created based on Fig. 8, respectively. Communication between the dependent interface DOFs belonging to different parts is unnecessary; thus, they are excluded (for example, 2 of part 1 and 2 of part 2).

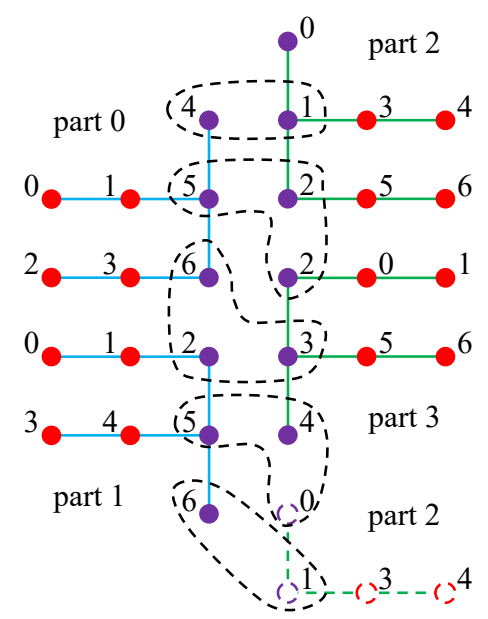

Fig. 11. Serial numbers of interface DOFs in each part of the rotating machine (step 1).
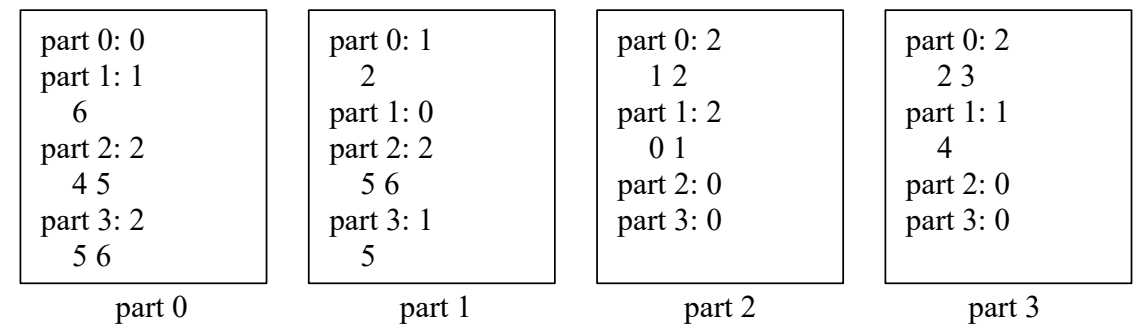

Fig. 12. Set of communication tables of step 1 (purple solid circles).

Next, the connection pattern of the interface DOFs after one time step is shown in Fig. 11. Here, the portion represented by the dotted line is the mesh of the moving body that protrudes upward, and is the portion in correspondence with the lower side of the stationary body based on the assumption in Fig. 5. The DOFs surrounded by the black dotted line represent the interface DOFs in a shared relationship. A set of communication tables (purple solid circles) created based on this connection pattern is shown in Fig. 12. In this manner, sets of communication tables (purple solid circles) are created for all of the connection patterns. In the example of this paper, four sets from the zeroth to the third are created.

In actual analysis, at each time step, the set of communication tables (red solid circles) and the set of communication tables (purple solid circles) of that time step are used. In this example, when the time step is $4 n+m(n=0,1,2, \cdots, m=0,1,2,3)$, interprocess communication is 


\section{Journal of Advanced Simulation in Science and Engineering}

performed using the set of communication tables (red solid circles) and the $m^{\text {th }}$ set of communication tables (purple solid circles).

We have proposed decomposing the meshes of the stationary and moving bodies independently, and letting the DOFs on the connection surface appearing on the surface of the subdomains be the interface DOFs shared between the parts. In the proposed method, there is no difference from the HDDM algorithm without a moving body, except that different sets of communication tables are used for each time step. When it is implemented, such tasks as loading and changing the sets of communication tables and updating the coordinate values of the moving body are added. They are very simple tasks; thus, it is easy to implement. Moreover, we expect that it will be possible to conduct the analysis of the moving bodies with high parallel efficiency in massively parallel environments. Furthermore, the proposed method is highly versatile, and we expect that if the HDDM can be applied, then the proposed method can be applied. Although this paper deals with three-dimensional electromagnetic field analysis as an example, we expect that it can be applied to structural analysis, heat transfer analysis, analysis in two dimensions, and various elements (tetrahedron/hexahedron, node element, edge element).

\section{Numerical examples}

\subsection{Model}
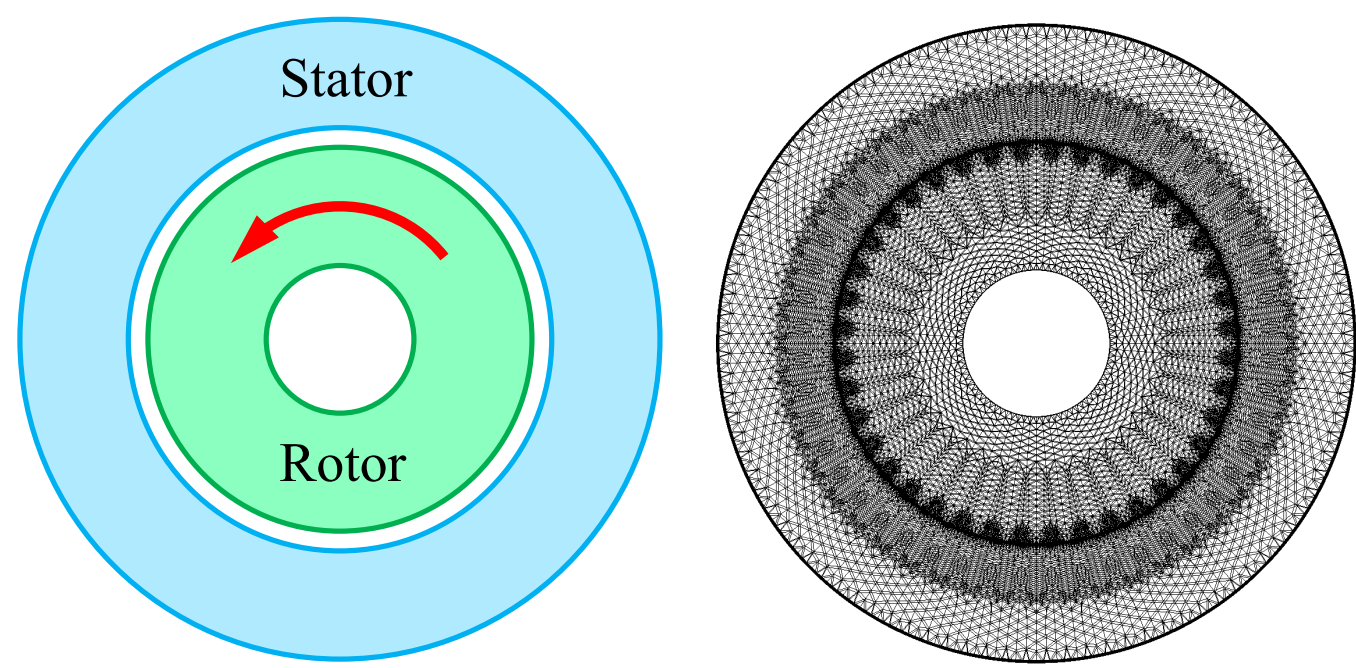

Fig. 13. Induction motor (Left: model, Right: mesh).

As a numerical example, a full model of a simplified rotating machine in imitation of an induction motor is considered (Fig. 13). The outside of the model is the stationary body (stator) and the inside is the moving body (rotor). The moving body rotates counterclockwise. The mesh of the stationary body has 2,903,040 elements, and 4,242,912 nodes, and that of the moving body has 2,808,960 elements, and 4,101,120 nodes. Time evolutionary analysis with the $A$ - $\phi$ method of a non-steady eddy current problem is performed. The total DOFs are 7,395,232. The connection surface between the stationary and moving bodies is constructed to enable the element 


\section{Journal of Advanced Simulation in Science and Engineering}

surfaces to coincide, even if the moving body moves, and there are 94,080 nodes on the connection surface.

In this paper, we use two models. One is a model combining the meshes of the stationary and moving bodies (Not moving). The other is a model decomposing the meshes of the stationary and moving bodies independently; thus, the moving body actually moves (Moving). Then, we compare the parallel performance.

\subsection{Strong scaling tests}

To measure the parallel performance, strong scaling tests are run on an Oakleaf-FX supercomputer [13] of Information Technology Center, The University of Tokyo, an FX100 supercomputer [14] of Information and Communications, Nagoya University, and an Oakforest-PACS supercomputer of JCAHPC [15]. The Oakleaf-FX supercomputer consists of 4,800 computer nodes of a Fujitsu PRIMEHPC FX10. Each node has 32 GB of memory and a Fujitsu SPARC64 IXfx processor with 16 cores. Nodes are connected with Tofu Interconnect and its bandwidth of interconnect is $5 \mathrm{~GB} / \mathrm{s} \times 2$. The FX100 supercomputer consists of 2,880 nodes of a Fujitsu PRIMEHPC FX100. Each node has 32 GB of memory, and a Fujitsu SPARC64 XIfx processor with 32 cores and 2 assistant cores that assist interprocess communications. Nodes are connected with Tofu Interconnect 2 and its bandwidth of interconnect is $12.5 \mathrm{~GB} / \mathrm{s} \times 2$. The Oakforest-PACS supercomputer consists of 8,208 computer nodes of a Fujitsu PRIMERGY CX1640 M1. Each node has 96 GB of memory and an Intel Xeon Phi 7250 processor with 68 cores. Nodes are connected with Intel Omni-Path and its bandwidth of interconnect is 12.5 $\mathrm{GB} / \mathrm{s}$.

Table 1. Numbers of parts and subdomains.

\begin{tabular}{|c|c|c|}
\hline Parts & $\begin{array}{c}\text { Not moving } \\
\text { parts } \times \text { subdomains }\end{array}$ & $\begin{array}{c}\text { Moving } \\
\text { parts } \times \text { subdomains } \\
\text { (Upper: Stationary body } \\
\text { Lower: Moving body) }\end{array}$ \\
\hline 6 & $6 \times 9,600$ & $\begin{array}{r}3 \times 9,600 \\
3 \times 9,600 \\
\end{array}$ \\
\hline 12 & $12 \times 4,800$ & $\begin{array}{l}6 \times 4,800 \\
6 \times 4,800 \\
\end{array}$ \\
\hline 24 & $24 \times 2,400$ & $\begin{array}{l}12 \times 2,400 \\
12 \times 2,400 \\
\end{array}$ \\
\hline 48 & $48 \times 1,200$ & $\begin{array}{l}24 \times 1,200 \\
24 \times 1,200 \\
\end{array}$ \\
\hline 96 & $96 \times 600$ & $\begin{array}{r}48 \times 600 \\
48 \times 600 \\
\end{array}$ \\
\hline 192 & $192 \times 300$ & $\begin{array}{l}96 \times 300 \\
96 \times 300 \\
\end{array}$ \\
\hline 384 & $384 \times 150$ & $\begin{array}{l}192 \times 150 \\
192 \times 150 \\
\end{array}$ \\
\hline
\end{tabular}




\section{Journal of Advanced Simulation in Science and Engineering}

Strong scaling tests are run with numbers of parts between six and 384. Domain decompositions are performed to ensure that the total number of subdomains is 57,600 (Table 1). In the case of Moving, half of the parts are allocated to each of the stationary and moving bodies. On the Oakleaf-FX supercomputer, one part is assigned to one computer node, and one MPI process is activated in each node. Each MPI process launches 16 threads. On the FX100 supercomputer, two parts are allocated to one node, and two MPI processes are activated in each node. Each MPI process launches 16 threads. On the Oakforest-PACS supercomputer, one part is assigned to one computer node, and one MPI process is activated in each node. Each MPI process launches 68 threads.

The CG method with simplified block diagonal scaling is applied to solve for interface DOFs. Subdomain problems are solved using the CG method with shifted incomplete Cholesky factorization (the shifted value is 1.2 , and the convergence criterion is $10^{-9}$ )

In the strong scaling tests, we fix the total computation amount and observe whether the elapsed time becomes shorter depending on the number of computer nodes. Therefore, in this study, the CG method for interface DOFs at each time step is uniformly stopped at 100 iterations, and 100 time steps are performed.

Table 2, 3 and 4 show the elapsed times, the parallel efficiencies based on the elapsed time of six parts, and the ratios of the elapsed time of Moving to that of Not moving. Figure 14 shows the parallel efficiencies and the relationship between number of parts and speed-up ratios. Here, "Theoretical" of the speed-up ratio means the theoretical value when the speed-up ratio of six parts is 1 , and when the number of parts being $x$ times corresponds with the speed-up ratio also being $x$.

On the Oakleaf-FX and FX100 supercomputers, the parallel efficiencies of Moving are above $90 \%$ up to 96 parts (1,536 cores), and the speed-up ratios are almost ideal up to 96 parts; thus, even in consideration of moving bodies, high parallel performance is obtained. Furthermore, the elapsed times of Moving show only an approximately 13 to $16 \%$ increase compared with those of Not moving.

On the Oakforest-PACS supercomputer, the parallel efficiencies of Moving are above $80 \%$ up to 48 parts (3,264 cores), and the speed-up ratios are almost ideal up to 48 parts; thus, even in consideration of moving bodies, high parallel performance is obtained, again. Furthermore, the elapsed times of Moving show only an approximately 16 to $20 \%$ increase compared with those of Not moving.

In terms of the number of cores, at more than 4,000 cores, the parallel performance is largely degraded, and the parallel efficiency becomes less than $70 \%$, on any supercomputer. For instance, in case of 384 parts on the Oakforest-PACS supercomputer, 68 cores handle 150 subdomains in each part; thus, the number of subdomains per core is too small and unequal. Therefore, it is guessed that the parallel performance is largely degraded. Meanwhile, at less than 4,000 cores, good parallel performance is obtained with this rotating machine model; thus, it is guessed that there is an upper limit of number of cores related to DOFs for good parallel performance. From Table 2, 3 and 4, it is guessed that above 30 subdomains per core are necessary to keep the parallel efficiency above $90 \%$. Regardless of the model, the number of elements per subdomain is constant in the HDDM; thus, it seems to mean that in rotating machine models with larger DOFs, it is possible to obtain better parallel performance even with larger number of parallel by the proposed method. 
Journal of Advanced Simulation in Science and Engineering

Here, supercomputers' bandwidth of interconnect per FLOPS of each node is much different (Oakleaf-FX 0.042283 B/s, FX100 0.022194 B/s, Oakforest-PACS 0.0041032 B/s). However, regardless of the architecture difference, the similar tendency is observed for any supercomputer. In the proposed method, communication is much less than the computation; thus, it is considered that the similar tendency is observed, regardless of the architecture difference. Furthermore, the tendency of parallel performance of Moving is similar to that of Not moving; thus, it is guessed that the proposed method inherits the characteristics of the parallel performance of the conventional method.

Table 5, 6 and 7 show the amounts of memory. Since Moving has different sets of communication tables for each time step, the amounts of memory are increasing by approximately $30 \%$.

Table 2. Elapsed times and parallel efficiencies (Oakleaf-FX).

\begin{tabular}{c|c|c|cc|cc|c}
\hline \hline Parts & Cores & $\begin{array}{c}\text { Subdomains } \\
\text { per core }\end{array}$ & $\begin{array}{c}\text { Time } \\
\text { [s] }\end{array}$ & $\begin{array}{c}\text { Not moving } \\
\text { Parallel } \\
\text { Efficiency [\%] }\end{array}$ & $\begin{array}{c}\text { Time } \\
\text { [s] }\end{array}$ & $\begin{array}{c}\text { Moving } \\
\text { Parallel } \\
\text { Efficiency [\%] }\end{array}$ & $\begin{array}{c}\text { Ratio [\%] } \\
\text { (Moving / } \\
\text { Not moving) }\end{array}$ \\
\hline 6 & 96 & 600 & 7,315 & - & 8,520 & - & 116.5 \\
12 & 192 & 300 & 3,637 & 101.0 & 4,231 & 101.3 & 116.3 \\
24 & 384 & 150 & 1,836 & 99.5 & 2,078 & 103.2 & 113.2 \\
48 & 768 & 75 & 950 & 95.8 & 1,072 & 99.2 & 112.9 \\
96 & 1,536 & 37.5 & 500 & 90.9 & 568 & 93.3 & 113.6 \\
192 & 3,072 & 18.75 & 300 & 74.7 & 330 & 80.3 & 108.6 \\
384 & 6,144 & 9.375 & 194 & 58.3 & 248 & 53.0 & 127.9 \\
\hline \hline
\end{tabular}

Table 3. Elapsed times and parallel efficiencies (FX100).

\begin{tabular}{c|c|c|cc|cc|c}
\hline \hline \multirow{2}{*}{ Parts } & Cores & $\begin{array}{c}\text { Subdomains } \\
\text { per core }\end{array}$ & $\begin{array}{c}\text { Time } \\
{[\mathrm{s}]}\end{array}$ & $\begin{array}{c}\text { Not moving } \\
\text { Efficiency [\%] }\end{array}$ & $\begin{array}{c}\text { Time } \\
{[\mathrm{s}]}\end{array}$ & $\begin{array}{c}\text { Moving } \\
\text { Parallel } \\
\text { Efficiency [\%] }\end{array}$ & $\begin{array}{c}\text { Ratio [\%] } \\
\text { (Moving / } \\
\text { Not moving) }\end{array}$ \\
\hline 6 & 96 & 600 & 5,130 & - & 6,155 & - & 120.0 \\
12 & 192 & 300 & 2,595 & 97.8 & 2,986 & 105.6 & 115.0 \\
24 & 384 & 150 & 1,335 & 94.9 & 1,508 & 102.6 & 112.9 \\
48 & 768 & 75 & 687 & 92.5 & 781 & 98.4 & 113.6 \\
96 & 1,536 & 37.5 & 381 & 83.2 & 421 & 90.8 & 110.5 \\
192 & 3,072 & 18.75 & 220 & 72.2 & 247 & 77.4 & 112.2 \\
384 & 6,144 & 9.375 & 152 & 51.9 & 166 & 57.4 & 108.8 \\
\hline \hline
\end{tabular}

Table 4. Elapsed times and parallel efficiencies (Oakforest-PACS).

\begin{tabular}{c|c|c|cc|cc|c}
\hline \hline \multirow{2}{*}{ Parts } & Cores & $\begin{array}{c}\text { Subdomains } \\
\text { per core }\end{array}$ & $\begin{array}{c}\text { Time } \\
{[\mathrm{s}]}\end{array}$ & $\begin{array}{c}\text { Not moving } \\
\text { Efficiency [\%] }\end{array}$ & $\begin{array}{c}\text { Time } \\
{[\mathrm{s}]}\end{array}$ & $\begin{array}{c}\text { Moving } \\
\text { Parallel } \\
\text { Efficiency [\%] }\end{array}$ & $\begin{array}{c}\text { Ratio [\%] } \\
\text { (Moving / } \\
\text { Not moving) }\end{array}$ \\
\hline 6 & 408 & 141.18 & 2,530 & - & 3,068 & - & 121.3 \\
12 & 816 & 70.588 & 1,320 & 92.3 & 1,582 & 94.4 & 119.9 \\
24 & 1,632 & 35.294 & 709 & 86.2 & 826 & 90.8 & 116.6 \\
48 & 3,264 & 17.647 & 386 & 79.7 & 456 & 82.2 & 118.1 \\
96 & 6,528 & 8.8235 & 246 & 62.3 & 299 & 62.2 & 121.5 \\
192 & 13,056 & 4.4118 & 171 & 44.7 & 220 & 42.0 & 128.6 \\
384 & 26,112 & 2.2059 & 160 & 23.7 & 196 & 23.5 & 122.3 \\
\hline \hline
\end{tabular}


Journal of Advanced Simulation in Science and Engineering
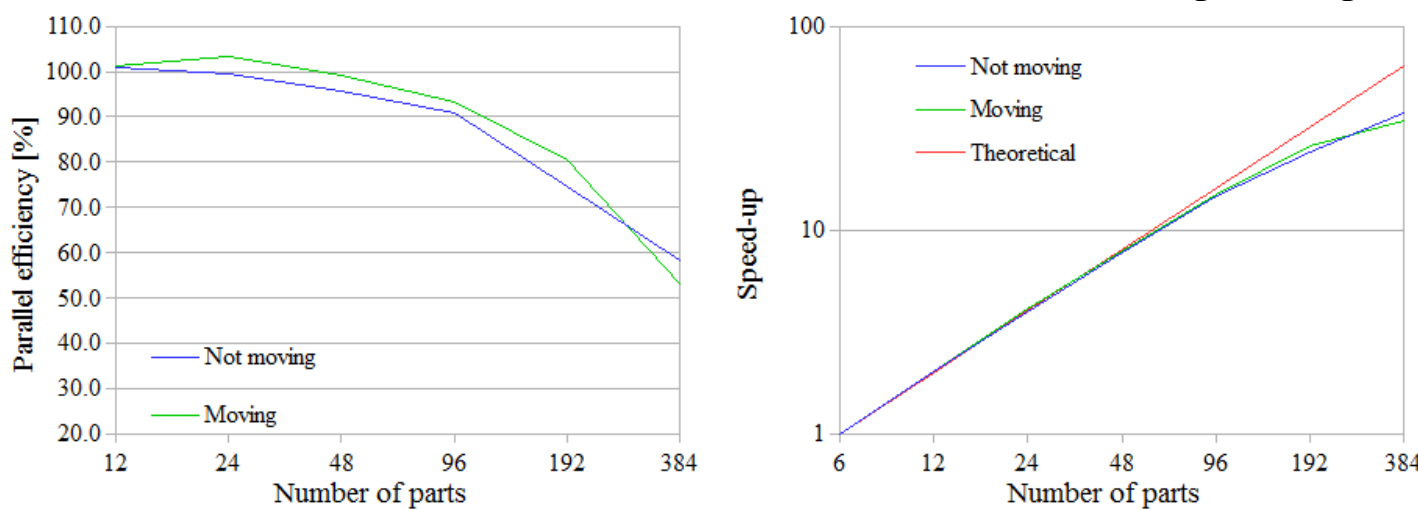

(a) Oakleaf-FX
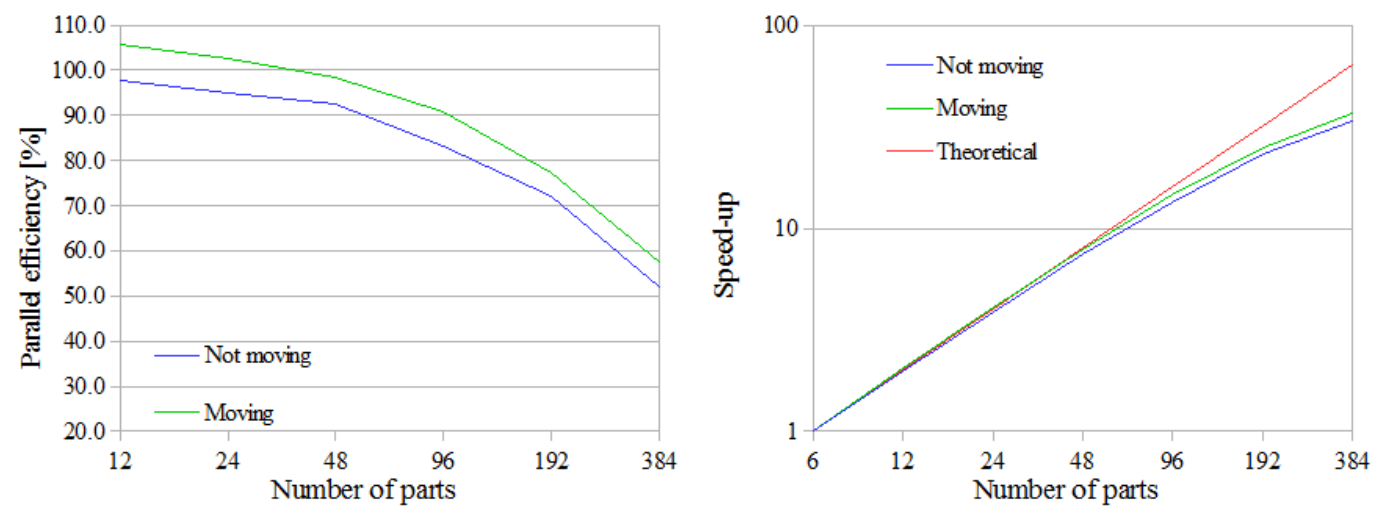

(b) FX100
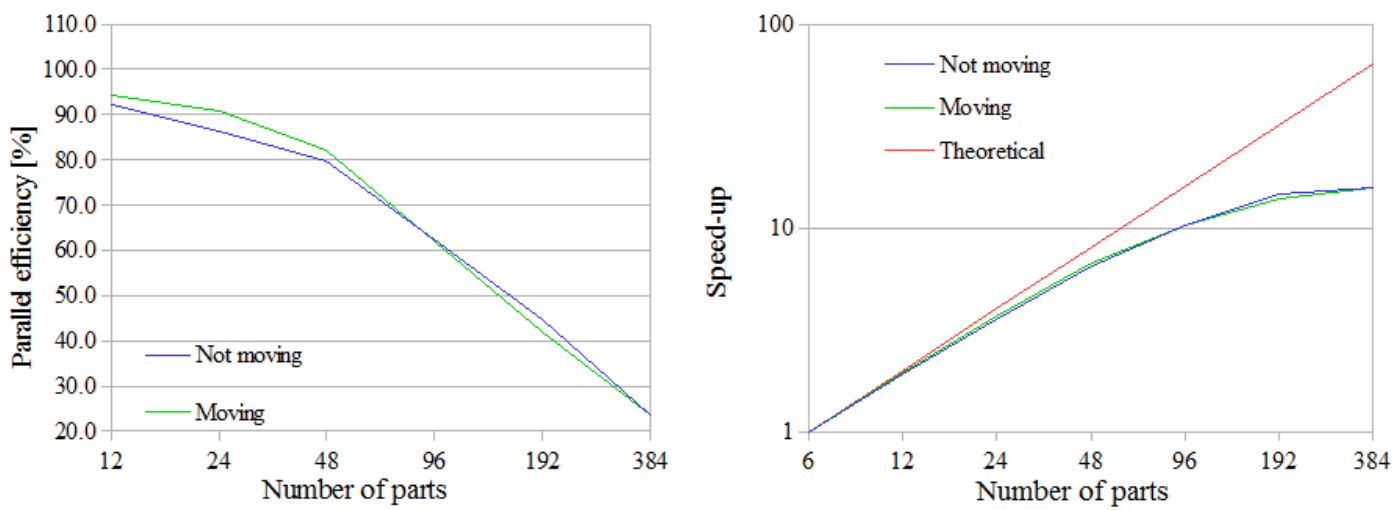

(c) Oakforest-PACS

Fig. 14. Parallel efficiency and speed-up ratio. 
Journal of Advanced Simulation in Science and Engineering

Table 5. Amounts of memory [GB] (Oakleaf-FX).

\begin{tabular}{c|c|c|c}
\hline \hline Parts & Not moving & Moving & $\begin{array}{c}\text { Ratio [\%] } \\
\text { (Moving/Not moving) }\end{array}$ \\
\hline 6 & 10.00 & 13.17 & 131.7 \\
12 & 10.01 & 13.18 & 131.7 \\
24 & 10.02 & 13.20 & 131.7 \\
48 & 10.04 & 13.23 & 131.8 \\
96 & 10.04 & 13.30 & 132.4 \\
192 & 10.05 & 13.72 & 136.6 \\
384 & 10.20 & 14.21 & 139.2 \\
\hline \hline
\end{tabular}

Table 6. Amounts of memory [GB] (FX100).

\begin{tabular}{c|c|c|c}
\hline \hline Parts & Not moving & Moving & $\begin{array}{c}\text { Ratio [\%] } \\
\text { (Moving/Not moving) }\end{array}$ \\
\hline 6 & 9.78 & 13.07 & 133.7 \\
12 & 9.79 & 13.08 & 133.6 \\
24 & 9.80 & 13.10 & 133.6 \\
48 & 9.82 & 13.13 & 133.7 \\
96 & 9.82 & 13.19 & 134.3 \\
192 & 9.82 & 13.62 & 138.6 \\
384 & 9.98 & 14.05 & 140.8 \\
\hline \hline
\end{tabular}

Table 7. Amounts of memory [GB] (Oakforest-PACS).

\begin{tabular}{c|c|c|c}
\hline \hline Parts & Not moving & Moving & $\begin{array}{c}\text { Ratio [\%] } \\
\text { (Moving/Not moving) }\end{array}$ \\
\hline 6 & 10.49 & 13.82 & 131.8 \\
12 & 10.51 & 13.84 & 131.7 \\
24 & 10.54 & 13.87 & 131.7 \\
48 & 10.57 & 13.92 & 131.7 \\
96 & 10.60 & 14.01 & 132.2 \\
192 & 10.63 & 14.47 & 136.2 \\
384 & 10.83 & 15.02 & 138.7 \\
\hline \hline
\end{tabular}

\subsection{Comparison with sequential computation}

To compare the computation time with conventional sequential computation, NEXST_Magnetic [16] is executed using one core of the Oakleaf-FX supercomputer. NEXST_Magnetic is software for sequential computation of the non-steady eddy current analysis using the finite element method, and it has been improved to analyze rotating machines. In this section, we let the iterative methods converge at each time step, and the simplified induction motor model is analyzed for 1,200 time steps. In the proposed method, computations are performed using 48 or 384 computer nodes of the Oakleaf-FX supercomputer.

The conventional sequential computation takes 12,561 seconds ( 3.49 hours) for five time steps. On the other hand, the proposed method using 48 nodes takes only 138 seconds $(0.0383$ hours) for five time steps, and 30,196 seconds ( 8.39 hours) for 1,200 time steps. From the ratio of computation times by the proposed method, we estimate that the conventional sequential computation takes over one month for 1,200 time steps. Furthermore, while parallel efficiency decreases, the proposed method using 384 nodes takes 5,776 seconds, that is, only approximately 1.60 hours for 1,200 time steps. Thus, the proposed method can significantly shorten 
the computation time compared with conventional sequential computation.

Figure 15 shows the number of iterations of the CG method for interface DOFs in each time step of the proposed method using 48 nodes. In the initial stage, when the non-linear computation is not stable, the number of iterations are large. However, after 150 time steps, the numbers of iterations stabilize around 300 iterations; thus, the proposed method can stably analyze the rotating machines.

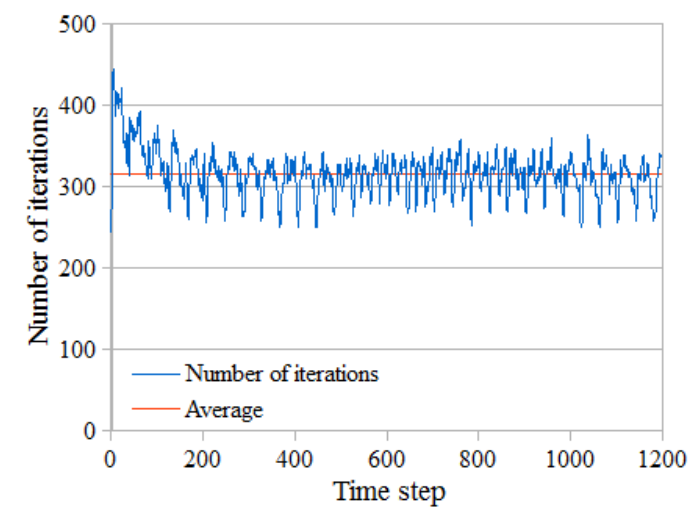

Fig. 15. Number of iterations in each time step.

\section{Conclusions}

To analyze devices including moving bodies efficiently on massively parallel computers, we have proposed the new domain decomposition technique for the HDDM. To prevent the DOFs on the connection surface from being located inside the subdomain, meshes of stationary and moving bodies are decomposed independently. Then, the connection surface appears on the surface of the subdomains; thus, the DOFs on the connection surface are treated as interface DOFs shared between parts. Furthermore, a set of communication tables relating to the original interface DOFs and sets of communication tables of the DOFs on the connection surface changing with time evolution are prepared separately. Then, they are used in combination at each time step. Finally, the proposed method has been implemented in the ADVENTURE_Magnetic, and the computational efficiency of the proposed method has been confirmed through analysis of a simplified induction motor model.

As a result, we have achieved efficient scalability on massively parallel computers. The parallel efficiencies of the proposed method have been above $90 \%$ up to 96 parts (1,536 cores), and the speed-up ratios have been almost ideal up to 96 parts on the Oakleaf-FX and FX100 supercomputers. On the Oakforest-PACS supercomputer, the parallel efficiencies of the proposed method have been above $80 \%$ up to 48 parts (3,264 cores), and the speed-up ratios have been almost ideal up to 48 parts; thus, even in consideration of moving bodies, high parallel performance has been obtained. Meanwhile, it has been guessed that above 30 subdomains per core are necessary to keep the parallel efficiency above $90 \%$. Moreover, the induction motor model with seven million DOF that is solved in more than one month using conventional sequential computation, has been successfully solved in only 1.60 hours using the proposed 


\section{Journal of Advanced Simulation in Science and Engineering}

method. The proposed method can significantly shorten the computation time compared with the conventional method. Meanwhile, in the proposed method, the elapsed times have increased by approximately $15 \%$, and amounts of memory have increased by approximately $30 \%$, compared with the case without a moving body. However, in the proposed method, the elapsed time decreases steadily as the number of computer nodes increases; thus, it is considered to be sufficiently practical.

\section{Acknowledgement}

This work is partially supported by JSPS KAKENHI Grant Number JP15K17499.

This work is partially supported by CREST, JST "Development of a Numerical Library based on Hierarchical Domain Decomposition for Post Petascale Simulation".

This work is partially supported by "Joint Usage/Research Center for Interdisciplinary Largescale Information Infrastructures" in Japan.

\section{References}

[1] Y. Takahashi, T. Tokumasu, M. Fujita, S. Wakao, K. Fujiwara, Y. Ishihara: Comparison between fast steady-state analysis methods for time-periodic nonlinear magnetic field problems, IEEE Transactions on Magnetics, Vol.48, No.2 (2012), pp.235-238.

[2] Y. Takahashi, T. Tokumasu, K. Fujiwara, T. Iwashita, H. Nakashima: Parallel TP-EEC method based on phase conversion for time-periodic nonlinear magnetic field problems, IEEE Transactions on Magnetics, Vol.51, No.3 (2015), 7001305.

[3] K. Miyata: Fast analysis method of time-periodic nonlinear fields, Journal of Math-forIndustry, Vol.3 (2011B-7) (2011), pp131-140.

[4] T. Nakano, Y. Kawase, T. Yamaguchi, M. Nakamura: Parallel computing of 3-D eddycurrent analysis with $A$ - $\phi$ method for rotating machines, IEEE Transactions on Magnetics, Vol.48, No.2 (2012), pp.975-978.

[5] Y. Takahashi, K. Fujiwara, T. Iwashita, H. Nakashima: Parallel finite-element analysis of rotating machines based on domain decomposition considering nonconforming mesh connection, IEEE Transactions on Magnetics, Vol.52, No.3 (2016), 7401604.

[6] S. Sugimoto, M. Ogino, H. Kanayama, S. Yoshimura: Introduction of a direct method at subdomains in non-linear magnetostatic analysis with HDDM, 2010 International Conference on Broadband, Wireless Computing, Communication and Applications (2010), pp.304-309.

[7] S. Sugimoto, D. Tagami, M. Ogino, A. Takei, H. Kanayama: Improvement of convergence in time-harmonic eddy current analysis by hierarchical domain decomposition method, The 20th International Conference on the Computation of Electromagnetic Fields, Montreal, 2015.

[8] S. Sugimoto, A. Takei, M. Ogino: Finite element analysis with tens of billions of degrees of freedom in a high-frequency electromagnetic field, Mechanical Engineering Letters, Vol.3 (2017), p.16-0067. 
[9] R. Glowinski, Q.V. Dinh, J. Periaux: Domain decomposition methods for nonlinear problems in fluid dynamics, Computer Methods in Applied Mechanics and Engineering, Vol.40, No.1 (1983), pp.27-109.

[10] A. Quarteroni and A. Valli: Domain Decomposition Methods for Partial Differential Equations, Clarendon Press, 1999.

[11] A. Toselli and O. Widlund: Domain Decomposition Methods: Algorithms and Theory (Springer Series in Computational Mechanics), Springer, 2004.

[12] Homepage of ADVENTURE Project: http://adventure.sys.t.u-tokyo.ac.jp/

[13] Homepage of Information Technology Center, The University of Tokyo: http://www.cc.u-tokyo.ac.jp/index-e.html

[14] Homepage of Super Computing System, Information and Communications, Nagoya University: http://www.icts.nagoya-u.ac.jp/en/sc/

[15] Homepage of JCAHPC: http://jcahpc.jp/eng/index.html

[16] Homepage of Frontier Simulation Software for Industrial Science: http://www.ciss.iis.u-tokyo.ac.jp/fsis/en/index.html 